\title{
THE WEDDERBURN PRINCIPAL THEOREM FOR A GENERALIZATION OF ALTERNATIVE ALGEBRAS
}

\author{
BY
}

HARRY F. SMITH

\begin{abstract}
A generalized alternative ring $I$ is a nonassociative ring $R$ in which the identities $(w x, y, z)+(w, x,[y, z])-w(x, y, z)-(w, y, z) x ;([w, x], y, z)+(w, x, y z)-y(w, x$, $z)-(w, x, y) z$; and $(x, x, x)$ are identically zero. It is here demonstrated that if $A$ is a finitedimensional algebra of this type over a field $F$ of characteristic $\neq 2,3$, then $A$ a nilalgebra implies $A$ is nilpotent.

A generalized alternative ring II is a nonassociative ring $\boldsymbol{R}$ in which the identities $(w x, y, z)+(w, x,[y, z])-w(x, y, z)-(w, y, z) x$ and $(x, y, x)$ are identically zero. Let $A$ be a finite-dimensional algebra of this type over a field $F$ of characteristic $\neq 2$. Then it is here established that (1) $A$ a nilalgebra implies $A$ is nilpotent; (2) $A$ simple with no nonzero idempotent other than 1 and $F$ algebraically closed imply $A$ itself is a field; and (3) the standard Wedderburn principal theorem is valid for $A$.
\end{abstract}

1. Preliminaries. Let $R$ be a nonassociative ring. As is customary, for $x, y, z \in R$ we denote by $(x, y, z)$ the associator $(x, y, z)=(x y) z-x(y z)$ and by $[x, y]$ the commutator $[x, y]=x y-y x$. A straightforward verification shows that the following identity, known as the Teichmüller identity, holds for all $w, x, y, z$ $\in R$ :

$$
(w x, y, z)-(w, x y, z)+(w, x, y z)=w(x, y, z)+(w, x, y) z .
$$

A nonassociative ring $R$ is called power-associative if for every $x \in R$ the subring generated by $x$ is associative.

A. Generalized alternative rings I. In [4] Kleinfeld defines a generalized alternative ring $\mathrm{I}$ to be a nonassociative ring $R$ such that for all $w, x, y, z \in R$ the following identities are satisfied:

$$
\begin{aligned}
(w x, y, z)+(w, x,[y, z]) & =w(x, y, z)+(w, y, z) x, \\
([w, x], y, z)+(w, x, y z) & =y(w, x, z)+(w, x, y) z, \\
(x, x, x) & =0 .
\end{aligned}
$$

That such a ring is power-associative can be readily verified as follows:

Received by the editors February 21, 1973.

AMS (MOS) subject classifications (1970). Primary 17A30; Secondary 17Al5.

Key words and phrases. Power-associative, noncommutative Jordan, generalized alternative rings I and II, alternative ring, finite-dimensional, nil, solvable, nilpotent, simple, degree one, algebraically closed field, nodal algebra, Albert decomposition, Peirce decomposition, Penico solvable, Wedderburn principal theorem, nil radical, separable. 
Theorem 1.1. A generalized alternative ring $I$ is power-associative.

Proof. Define $x^{n}=x^{n-1} x$. We need to show $x^{i} x^{j}=x^{i+j}$ for any $i, j>0$. From (1.3) we have $x^{3}=x^{2} x=x x^{2}$. Also, (1.1) and (1.3) yield $\left(x^{2}, x, x\right)+(x, x,[x, x])$ $=x(x, x, x)+(x, x, x) x$ or $\left(x^{2}, x, x\right)=0$, which implies $x^{4}=x^{3} x=x^{2} x^{2}$; while (1.2) and (1.3) yield $([x, x], x, x)+\left(x, x, x^{2}\right)=x(x, x, x)+(x, x, x) x$ or $\left(x, x, x^{2}\right)$ $=0$, which implies $x^{2} x^{2}=x x^{3}$.

The proof is now by induction. We assume $x^{i} x^{j}=x^{i+j}$ for $i+j<n ; i, j>0$ and $n>4$. Then (1.1) gives $\left(x^{2}, x^{n-2-i}, x^{i}\right)+\left(x, x,\left[x^{n-2-i}, x^{i}\right]\right)=x\left(x, x^{n-2-i}, x^{i}\right)$ $+\left(x, x^{n-2-i}, x^{i}\right) x$ or $\left(x^{2}, x^{n-2-i}, x^{i}\right)=0$, using the induction assumption. Thus, except possibly for $i=n-1, x^{n-i} x^{i}=x^{2} x^{n-2}$. But, again using the induction assumption, (1.1) gives $\left(x^{2}, x^{n-3}, x\right)+\left(x, x,\left[x^{n-3}, x\right]\right)=x\left(x, x^{n-3}, x\right)+\left(x, x^{n-3}\right.$, $x) x$ or $\left(x^{2}, x^{n-3}, x\right)=0$, that is $x^{n}=x^{n-1} x=x^{2} x^{n-2}$. Thus $x^{n-i} x^{i}=x^{n}$, except possibly for $i=n-1$. Finally, (1.2) and the induction assumption yield $\left(\left[x, x^{n-3}\right], x, x\right)+\left(x, x^{n-3}, x^{2}\right)=x\left(x, x^{n-3}, x\right)+\left(x, x^{n-3}, x\right) x$ or $\left(x, x^{n-3}, x^{2}\right)=0$, that is $x x^{n-1}=x^{n-2} x^{2}=x^{n}$. This completes the induction.

Let $R$ be a generalized alternative ring $I$. If one defines a new multiplication for $R$ by $x * y=y x$, then a straightforward verification shows that under this new multiplication identity (1.1) is converted to identity (1.2) and vice versa. Thus, since identity (1.3) is left unchanged, the resulting ring is itself a generalized alternative ring $I$. We henceforth refer to this procedure as passing to the anti-isomorphic copy of $R$.

In this work we consider generalized alternative algebras I over fields of characteristic $\neq 2$, 3. In addition to the above defining identities, we also make repeated use of the following:

$$
\begin{gathered}
(y, x, x)+(x, y, x)+(x, x, y)=0, \\
(w, x y, z)-(w, x, z y)+(w, x, y) z-(w, y, z) x=0, \\
(z, y x, w)-(y z, x, w)+z(y, x, w)-x(z, y, w)=0, \\
(x, x, y x)=(x, x, y) x, \\
(x, x y, x)=x(x, y, x), \\
\left(x^{2}, y, x\right)=2 x(x, y, x), \\
\left(x^{2}, x, y\right)=\left(x, x^{2}, y\right)=2(x, x, y x) .
\end{gathered}
$$

Identity (1.4) is obtained from linearization of (1.3). Identity (1.5) is obtained by subtracting (T) from (1.1), after which (1.6) follows from (1.5) by passing to the anti-isomorphic copy of $R$. Identities (1.7), (1.8), and (1.9) are established in [4]. To see that $\left(x^{2}, x, w\right)=\left(x, x^{2}, w\right)$, we let $z=y=x$ in (1.6); while to see that $\left(x, x^{2}, z\right)=2(x, x, z x)$, we let $w=y=x$ in (1.5) and then apply (1.3) and (1.7). Now taking $w, z$ to be $y$, these last two equations together give (1.10).

B. Generalized alternative rings II. A generalized alternative ring II is defined 
by Kleinfeld in [5] to be a nonassociative ring $R$ such that for all $w, x, y, z \in R$ the following identities are satisfied:

$$
\begin{aligned}
(w x, y, z)+(w, x,[y, z]) & =w(x, y, z)+(w, y, z) x, \\
(x, y, x) & =0 .
\end{aligned}
$$

From these identities one easily generates:

$$
\begin{gathered}
(x, y, z)=-(z, y, x), \\
(w, x y, z)-(w, x, z y)+(w, x, y) z-(w, y, z) x=0, \\
(w x, z, z)=w(x, z, z)+(w, z, z) x, \\
\left(z, x, z^{2}\right)=0, \\
z(z, z, x)=(z, z, z x)=(z, z, x z)=(z, z, x) z .
\end{gathered}
$$

Identity (2.3) follows from linearization of (2.2). Identity (2.4) is obtained by subtracting (T) from (2.1). If one takes $y=z$ in (2.1), one obtains (2.5). Letting $w=y=z$ in (2.4) and applying (2.2), one obtains (2.6).

To see that $z(z, z, x)=(z, z, z x)$, we let $w=y=z$ in (2.1) and then apply (2.2) and (2.3). To see that $(z, z, z x)=(z, z, x) z$, we take $w, x, z$ to be $z$ and $y$ to be $x$. in (2.4) and then apply (2.2). Finally, to see that $(z, z, x) z=(z, z, x z)$, we take $x$, $y, z$ to be $z$ and $w$ to be $x$ in (2.1) and then apply (2.2) and (2.3). This establishes (2.7).

A nonassociative ring which satisfies identities (2.2) and (2.6) is called noncommutative Jordan. From [10] an algebra of this type over a field of characteristic $\neq 2$ is known to be power-associative.

2. Finite-dimensional nilalgebras. Let $A$ be a power-associative algebra. An element $x \in A$ is said to be nilpotent if there exists an integer $k>0$ for which $x^{k}=0$. Should every element of the algebra $A$ be nilpotent, then $A$ is called a nilalgebra. For any algebra $A$ one obtains a derived series of subalgebras $A^{(0)} \supseteq A^{(1)} \supseteq \cdots$ by defining inductively $A^{(0)}=A, A^{(i+1)}=\left(A^{(i)}\right)^{2} . A$ is called solvable in case $A^{(m)}=0$ for some integer $m>0$. A nonassociative algebra $A$ is called nilpotent in case there exists an integer $n>0$ such that any product $x_{1} x_{2} \cdots x_{n}$ of $n$ elements $x_{i} \in A$, no matter how associated, is zero.

For an algebra $A$ and $x \in A$, the linear operators on $A$ of right and left multiplication by $x$ are denoted by $R_{x}$ and $L_{x}$, respectively. Let $M(A)$ denote the subalgebra generated by all right and left multiplications of $A$ in the associative algebra of all linear operators on $A$. If $B$ is any subset of $A$, we shall write $B^{*}$ for the subalgebra of $M(A)$ generated by all right and left multiplications of $A$ which correspond to elements of $B$. 


\section{A. Generalized alternative algebras I.}

Lemma 1.1. Let $B$ be a generalized alternative algebra $I$ over a field $F$ of characteristic $\neq 2$, 3. Suppose $B=F b+C$ where $C$ is a subalgebra of $B$ such that $B^{2} \subseteq C$. If $H=B^{*} C^{*}+C^{*}$, then $S_{x} S_{y} S_{z} \in H$ for $S=L$ or $R$ and all $x, y, z$ $\in B$.

Proof. We begin by making some reductions. First, since every product of three operators of right or left multiplication corresponding to elements of $B=F b$ $+C$ may be expressed as a linear combination of products $S_{x} S_{y} S_{z}$ where each of $x, y, z$ is either in $C$ or equal to $b$, it suffices to verify only that products of this latter form belong to $H$. In particular, since $c \in C$ clearly implies $S_{x} S_{y} S_{c} \in H$, we need only consider products of the form $S_{x} S_{y} S_{b}$ where each of $x$ and $y$ is either in $C$ or equal to $b$.

Secondly, should $S_{x_{1}} S_{x_{2}} S_{x_{3}} \in H$, then by passing to the anti-isomorphic copy of $B$ one sees that $S_{x_{1}}^{\prime} S_{x_{2}}^{\prime} S_{x_{3}}^{\prime} \in H$, where $S_{x_{i}}^{\prime}=R_{x_{i}}\left(L_{x_{i}}\right)$ if $S_{x_{i}}=L_{x_{i}}\left(R_{x_{i}}\right)$ for $i=1,2,3$.

Henceforth let $c, c^{\prime} \in C$. From (1.1), $(w c, y, b)+(w, c,[y, b])=w(c, y, b)+(w$, $y, b) c$, we have $R_{c} R_{y} R_{b}-R_{c} R_{b y}+R_{c(b y)}=R_{(c y) b}+R_{y} R_{b} R_{c}-R_{y b} R_{c}$. Since by assumption $B^{2} \subseteq C$, this yields $R_{c} R_{y} R_{b} \in H$ or

(1.a) $R_{c} R_{c} R_{b}, R_{c} R_{b} R_{b} \in H$.

Now applying our second reduction, (1.a) in turn yields

(1.b) $L_{c} L_{c} L_{b}, L_{c} L_{b} L_{b} \in H$.

From (1.2), $([b, x], y, c)+(b, x, y c)=y(b, x, c)+(b, x, y) c$, we have $-L_{x b} R_{c}$ $+R_{c} L_{x b}-R_{c} L_{x} L_{b}=R_{(b, x, c)}-L_{x} L_{b} R_{c}$. Again using the assumption $B^{2} \subseteq C$, as we will continually do throughout, this yields $R_{c} L_{x} L_{b} \in H$ or

(1.c) $R_{c} L_{c}{ }^{\prime} L_{b}, R_{c} L_{b} L_{b} \in H$.

Again applying our second reduction, as we also will continually do throughout, (1.c) in turn yields

(1.d) $L_{c} R_{c} \cdot R_{b}, L_{c} R_{b} R_{b} \in H$.

From (1.1), $\left(c^{\prime} b, y, c\right)+\left(c^{\prime}, b,[y, c]\right)=c^{\prime}(b, y, c)+\left(c^{\prime}, y, c\right) b$, using (1.d) we now obtain

(1.e) $R_{c} L_{c} R_{b}, L_{c} R_{c} L_{b} \in H$.

Adding (1.1), $\left(b x, c, c^{\prime}\right)+\left(b, x,\left[c, c^{\prime}\right]\right)=b\left(x, c, c^{\prime}\right)+\left(b, c, c^{\prime}\right) x$, to (1.2), ([b, x], $\left.c^{\prime}, c\right)+\left(b, x, c^{\prime} c\right)=c^{\prime}(b, x, c)+\left(b, x, c^{\prime}\right) c$, we have

(1.f) $R_{c} R_{c^{\prime}} L_{b}, L_{c} L_{c} R_{b} \in H$.

Linearization of $(1.10),\left(b^{2}, b, y\right)=2(b, b, y b)$, gives

$$
\left(b^{2}, c, y\right)+(b c, b, y)+(c b, b, y)=2[(b, b, y c)+(b, c, y b)+(c, b, y b)] .
$$

Using (1.c) this yields $2 R_{b} L_{c} L_{b} \in H$ or

(1.g) $R_{b} L_{c} L_{b}, L_{b} R_{c} R_{b} \in H$.

From (1.6) we obtain $(z, b c, b)-(b z, c, b)+z(b, c, b)-c(z, b, b)=0$. Using (1.g) this implies $R_{b c} R_{b} \in H$. Then from (1.1), $(w b, b, c)+(w, b,[b, c])=w(b, b$, $c)+(w, b, c) b$, we have 
(1.h) $R_{b} R_{c} R_{b}, L_{b} L_{c} L_{b} \in H$.

Next from (1.5), $(b, c y, b)-(b, c, b y)+(b, c, y) b-(b, y, b) c=0$, if we use (1.h) we obtain $L_{b c} R_{b}-L_{c} R_{b} L_{b} \in H$. Since from (1.6), $(c, b x, b)-(b c, x, b)+c(b, x$, b) $-x(c, b, b)=0$, one has $L_{b} L_{c} R_{b}-L_{b c} R_{b} \in H$, adding this to $L_{b c} R_{b}$ $-L_{c} R_{b} L_{b}$ gives

(i.1) $L_{b} L_{c} R_{b}-L_{c} R_{b} L_{b} \in H$.

From (1.4), $(c x, b, b)+(b, c x, b)+(b, b, c x)=0$, using (1.b) and (1.d) one also has

(i.2) $L_{c} R_{b} L_{b}-L_{c} L_{b} R_{b} \in H$.

If we now linearize $(1.8)$ to obtain $(c, b y, b)+(b, c y, b)+(b, b y, c)=c(b, y, b)$ $+b(c, y, b)+b(b, y, c)$, then using (1.c), (1.g), (i.1), and (i.2) we have $2 L_{b} R_{c} L_{b}$ $\in H$ or

(1.i) $L_{b} R_{c} L_{b}, R_{b} L_{c} R_{b} \in H$.

From linearization of (1.4) we obtain $(b x, b, c)+(b, c, b x)+(c, b x, b)+(b x, c$, $b)+(c, b, b x)+(b, b x, c)=0$. Using (1.g), (1.h), and (1.i) this yields

(1.j) $L_{b} L_{c} R_{b}, R_{b} R_{c} L_{b} \in H$.

From (1.1), $\left(b^{2}, y, c\right)+(b, b,[y, c])=b(b, y, c)+(b, y, c) b$, using (1.b), (1.g), and (1.i) we have

(1.k) $R_{c} L_{b} R_{b}, L_{c} R_{b} L_{b} \in H$.

Next (1.4), $(x c, b, b)+(b, x c, b)+(b, b, x c)=0$, together with (1.a), (1.c), and (1.k) gives

(1.m) $R_{c} R_{b} L_{b}, L_{c} L_{b} R_{b} \in H$.

From (1.10), $\left(b^{2}, b, y\right)=2(b, b, y b)$, we obtain $2 R_{b} L_{b} L_{b} \in H$, that is (1.n) $R_{b} L_{b} L_{b}, L_{b} R_{b} R_{b} \in H$.

We next add (1.1), $\left(b^{2}, x, b\right)+(b, b,[x, b])=b(b, x, b)+(b, x, b) b$, to (1.2), $([b, x], b, b)+\left(b, x, b^{2}\right)=b(b, x, b)+(b, x, b) b$ to derive $\left(b^{2}, x, b\right)+\left(b, x, b^{2}\right)$ $+(b, b,[x, b])+([b, x], b, b)=2 b(b, x, b)+2(b, x, b) b$. Since $(1.9)$ gives $2 b(b, x, b)$ $=\left(b^{2}, x, b\right)$, and since by passing to the anti-isomorphic copy of $B$ this in turn gives $2(b, x, b) b=\left(b, x, b^{2}\right)$, our last equation simplifies to $(b, b,[x, b])+([b, x], b$, $b)=0$. Then using $(1.6),(x b, b, b)=(b, x b, b)+b(x, b, b)-b(b, x, b)$, we have $(b, b,[x, b])+(b x, b, b)=(b, x b, b)+b(x, b, b)-b(b, x, b)$. Now by passing to the anti-isomorphic copy of $B,(1.8)$ and $(1.7)$ become $(b, x b, b)=(b, x, b) b$ and $b(x, b, b)=(b x, b, b)$, respectively. Hence our equation again simplifies, this time to $(b, b,[x, b])=[(b, x, b), b]$. Using (1.n) this gives

(p.1) $L_{b} L_{b} L_{b}+R_{b} L_{b} R_{b}+L_{b} R_{b} L_{b} \in H$.

From (1.4), $(b x, b, b)+(b, b, b x)+(b, b x, b)=0$, using (1.8) we obtain $(b x, b, b)$ $=-(b, b, b x)-b(b, x, b)$. If we use (1.n), this yields

(p.2) $L_{b} L_{b} L_{b}-L_{b} R_{b} L_{b} \in H$.

Now subtracting (p.2) from (p.1) we have

(p.3) $R_{b} L_{b} R_{b}+2 L_{b} R_{b} L_{b} \in H$.

Next from linearization of (1.4) we obtain $\left(x, b, b^{2}\right)+\left(b, b^{2}, x\right)+\left(b^{2}, x, b\right)$ $+\left(x, b^{2}, b\right)+\left(b^{2}, b, x\right)+\left(b, x, b^{2}\right)=0$ or $2\left(x, b, b^{2}\right)+2\left(b^{2}, b, x\right)+\left(b^{2}, x, b\right)$ $+\left(b, x, b^{2}\right)=0$, since $(1.10)$ implies $\left(b, b^{2}, x\right)=\left(b^{2}, b, x\right)$, and since by passing to 
the anti-isomorphic copy of $B$ this in turn implies $\left(x, b^{2}, b\right)=\left(x, b, b^{2}\right)$. Thus we have

(p.4) $\left(L_{b^{2}}\right) R_{b}-\left(R_{b^{2}}\right) L_{b} \in H$.

Now (1.9) gives $\left(b^{2}, x, b\right)=2 b(b, x, b)$, and by passing to the anti-isomorphic copy of $B$ this in turn gives $\left(b, x, b^{2}\right)=2(b, x, b) b$. Hence using (1.n) we also have

(p.5) $2 L_{b} R_{b} L_{b}-\left(L_{b^{2}}\right) R_{b} \in H$, and

(p.6) $2 R_{b} L_{b} R_{b}-\left(R_{b^{2}}\right) L_{b} \in H$.

If we now subtract (p.4) and (p.5) from (p.6), we obtain

(p.7) $2 R_{b} L_{b} R_{b}-2 L_{b} R_{b} L_{b} \in H$.

Lastly, adding (p.7) to (p.3) we have $3 R_{b} L_{b} R_{b} \in H$ or

(1.p) $R_{b} L_{b} R_{b}, L_{b} R_{b} L_{b} \in H$.

In addition, (p.2) and (1.p) together also show

(1.q) $L_{b} L_{b} L_{b}, R_{b} R_{b} R_{b} \in H$.

Finally, by passing to the anti-isomorphic copy of $B$, from (1.8) we obtain as before $(b, x b, b)=(b, x, b) b$. Using (1.n) and (1.p) we then have

(1.r) $R_{b} R_{b} L_{b}, L_{b} L_{b} R_{b} \in H$.

This completes the proof of the lemma.

From Schafer's proof of Theorem 3 in [15], which proof in turn is modelled on that of Albert for standard algebras in [2], it follows that Lemma 1.1 is sufficient to obtain the following result.

Theorem 1.2. Let $A$ be a finite-dimensional generalized alternative algebra I over a field $F$ of characteristic $\neq 2,3$. If $B$ is a solvable subalgebra of $A$, then $B^{*}$ is nilpotent.

Lemma 1.2. Let $A$ be a generalized alternative algebra I over a field $F$ of characteristic $\neq 2,3$; and let $B$ be a subalgebra of $A$. If $x \in A$ is such that $x B \subseteq B, B x \subseteq B$, then $\left(x^{2} B\right) B \subseteq B, x^{2} B^{2} \subseteq B, B\left(x^{2} B\right) \subseteq B, B^{2} x^{2} \subseteq B$, and $\left(x^{2} B\right)^{2} B \subseteq B$.

Proof. We assume throughout that $b_{i} \in B$ for $i=1$, 2, 3. From (1.5) we have $\left(x, b_{1} x, b_{2}\right)-\left(x, b_{1}, b_{2} x\right)+\left(x, b_{1}, x\right) b_{2}-\left(x, x, b_{2}\right) b_{1}=0$ or $\left(x^{2} B\right) B \subseteq B$. Then from (1.5) we also have $\left(x, x b_{1}, b_{2}\right)-\left(x, x, b_{2} b_{1}\right)+\left(x, x, b_{1}\right) b_{2}-\left(x, b_{1}, b_{2}\right) x=0$ or $x^{2} B^{2} \subseteq B$. Next (1.6) gives $\left(x, x b_{2}, b_{1}\right)-\left(x^{2}, b_{2}, b_{1}\right)+x\left(x, b_{2}, b_{1}\right)-b_{2}\left(x, x, b_{1}\right)$ $=0$ or $B\left(x^{2} B\right) \subseteq B$. Now (1.6) also gives $\left(b_{1}, x b_{2}, x\right)-\left(x b_{1}, b_{2}, x\right)+b_{1}\left(x, b_{2}, x\right)$ $-b_{2}\left(b_{1}, x, x\right)=0$ or $B\left(B x^{2}\right) \subseteq B$, whence (1.6) yields $\left(b_{1}, b_{2} x, x\right)-\left(b_{2} b_{1}, x, x\right)$ $+b_{1}\left(b_{2}, x, x\right)-x\left(b_{1}, b_{2}, x\right)=0$ or $B^{2} x^{2} \subseteq B$.

There remains only to show $\left(x^{2} B\right)^{2} B \subseteq B$. We first observe that from (1.5) we have $\left(b_{1}, b_{2} x, x\right)-\left(b_{1}, b_{2}, x^{2}\right)+\left(b_{1}, b_{2}, x\right) x-\left(b_{1}, x, x\right) b_{2}=0$ or $\left(B x^{2}\right) B \subseteq B$. Also, (1.6) then implies $\left(x^{2}, b_{1}\left(b_{2} x^{2}\right), b_{3}\right)-\left(b_{1} x^{2}, b_{2} x^{2}, b_{3}\right)+x^{2}\left(b_{1}, b_{2} x^{2}, b_{3}\right)$ $-\left(b_{2} x^{2}\right)\left(x^{2}, b_{1}, b_{3}\right)=0$ or $\left(B x^{2}\right)^{2} B \subseteq B$. Now using (1.4), (1.1) gives

$$
\begin{gathered}
{\left[\left(x^{2} b_{1}\right) b_{2}\right] x^{2}-\left(x^{2} b_{1}\right)\left(x^{2} b_{2}\right)+x^{2}\left[b_{1}\left(x^{2} b_{2}\right)\right]-x^{2}\left[\left(b_{1} b_{2}\right) x^{2}\right]} \\
=\left(x^{2}, b_{2}, x^{2}\right) b_{1}=-\left(b_{2}, x^{2}, x^{2}\right) b_{1}-\left(x^{2}, x^{2}, b_{2}\right) b_{1} ;
\end{gathered}
$$


and (1.2) gives

$$
\begin{gathered}
-\left[\left(b_{1} x^{2}\right) b_{2}\right] x^{2}+\left(b_{1} x^{2}\right)\left(b_{2} x^{2}\right)-x^{2}\left[b_{1}\left(b_{2} x^{2}\right)\right]+\left[x^{2}\left(b_{1} b_{2}\right)\right] x^{2} \\
=b_{2}\left(x^{2}, b_{1}, x^{2}\right)=-b_{2}\left(x^{2}, x^{2}, b_{1}\right)-b_{2}\left(b_{1}, x^{2}, x^{2}\right) .
\end{gathered}
$$

Adding these last two equations and using (1.1), (1.2), and (1.4), we have

$$
\begin{aligned}
& {\left[\left(x^{2} b_{1}\right) b_{2}\right] x^{2}-\left(x^{2} b_{1}\right)\left(x^{2} b_{2}\right)+x^{2}\left[b_{1}\left(x^{2} b_{2}\right)\right]-x^{2}\left[\left(b_{1} b_{2}\right) x^{2}\right] } \\
&-\left[\left(b_{1} x^{2}\right) b_{2}\right] x^{2}+\left(b_{1} x^{2}\right)\left(b_{2} x^{2}\right)-x^{2}\left[b_{1}\left(b_{2} x^{2}\right)\right]+\left[x^{2}\left(b_{1} b_{2}\right)\right] x^{2} \\
&=-b_{2}\left(b_{1}, x^{2}, x^{2}\right)-\left(b_{2}, x^{2}, x^{2}\right) b_{1}-b_{2}\left(x^{2}, x^{2}, b_{1}\right)-\left(x^{2}, x^{2}, b_{2}\right) b_{1} \\
&=-\left(b_{2} b_{1}, x^{2}, x^{2}\right)-\left(x^{2}, x^{2}, b_{2} b_{1}\right) \\
&=\left(x^{2}, b_{2} b_{1}, x^{2}\right) .
\end{aligned}
$$

Finally, multiplication of this last equation on the right by $b_{3}$ yields $\left(x^{2} B\right)^{2} B$ $\subseteq B$.

Lemma 1.3. Let $A$ be a generalized alternative algebra I over a field $F$ of characteristic $\neq 2,3$; and let $B$ be a subspace of $A$. If $x \in A$ is such that $x B \subseteq B, B x \subseteq B, x^{2} B \subseteq B$, then $x^{k} B \subseteq B, B x^{k} \subseteq B$ for $k=1,2,3, \ldots$

Proof. Let $b \in B$. We note that $(1.4),(b, x, x)+(x, b, x)+(x, x, b)=0$, implies $B x^{2} \subseteq B$. Hence we have $x^{k} B \subseteq B, B x^{k} \subseteq B$ for $k=1,2$. The proof now is by induction. We assume $x^{k} B \subseteq B, B x^{k} \subseteq B$ for $k\langle n, n>2$. From (1.10) and (1.7) we have $\left(x, x^{2}, y\right)=2(x, x, y) x$. Linearization of this identity gives

$$
\begin{aligned}
& \left(x^{n-2}, x^{2}, b\right)+\left(x, x^{n-2} x, b\right)+\left(x, x x^{n-2}, b\right) \\
& \quad=2\left[\left(x^{n-2}, x, b\right) x+\left(x, x^{n-2}, b\right) x+(x, x, b) x^{n-2}\right] .
\end{aligned}
$$

Applying the induction assumption, we now have $3 x^{n} b \in B$ or $x^{n} B \subseteq B$. Next linearization of (1.4) gives $\left(b, x, x^{n-1}\right)+\left(b, x^{n-1}, x\right)+\left(x, b, x^{n-1}\right)+\left(x^{n-1}, b, x\right)$ $+\left(x, x^{n-1}, b\right)+\left(x^{n-1}, x, b\right)=0$. Again applying the induction assumption, we have $2 b x^{n} \in B$ or $B x^{n} \subseteq B$, and our induction is complete.

The proof of the following theorem is now the same as that of Theorem 4 in [15], with the one exception that, since a generalized alternative algebra $I$ is not necessarily noncommutative Jordan, we need to make use of our Lemma 1.3 in addition to Theorem 1.2 and Lemma 1.2 above.

Theorem 1.3. Let $A$ be a finite-dimensional generalized alternative algebra $\mathrm{I}$ over a field $F$ of characteristic $\neq 2$, 3. If $A$ is a nilalgebra, then $A$ is nilpotent.

B. Generalized alternative algebras II.

Lemma 2.1. Let $B$ be a generalized alternative algebra II over a field $F$ of characteristic $\neq 2$. Suppose $B=F b+C$ where $C$ is a subalgebra of $B$ such that 
$B^{2} \subseteq C$. If $H=B^{*} C^{*}+C^{*}$, then $S_{x} S_{y} S_{z} \in H$ for $S=L$ or $R$ and all $x, y, z$ $\in B$.

Proof. As in the proof of Lemma 1.1, it suffices to verify only that $H$ contains products of the form $S_{x} S_{y} S_{b}$ where each of $x$ and $y$ is either in $C$ or equal to $b$. In addition, we note that (2.2) implies $L_{b} R_{b}=R_{b} L_{b}$.

Throughout we assume $c, c^{\prime} \in C$. From (2.1), $(w c, y, b)+(w, c,[y, b])=w(c$, $y, b)+(w, y, b) c$, we have $R_{c} R_{y} R_{b}-R_{c} R_{b y}+R_{c(b y)}=R_{(c y) b}+R_{y} R_{b} R_{c}-R_{y b} R_{c}$. Since by assumption $B^{2} \subseteq C$, this last equation yields $R_{c} R_{y} R_{b} \in H$ or

(2.a) $R_{c} R_{c^{\prime}} R_{b}, R_{c} R_{b} R_{b} \in H$.

From $(2.1),(c x, y, b)+(c, x,[y, b])=c(x, y, b)+(c, y, b) x$, we also obtain $L_{c} R_{y} R_{b}-L_{c} R_{b y}+R_{b y} L_{c}=R_{y} R_{b} L_{c}+L_{(c, y, b)}$. Again using the assumption $B^{2}$ $\subseteq C$, as we will continually do throughout, this yields $L_{c} R_{y} R_{b} \in H$ or

(2.b) $L_{c} R_{c} \cdot R_{b}, L_{c} R_{b} R_{b} \in H$.

Now (2.3), $\quad(x c, y, b)=-(b, y, x c), \quad$ gives $\quad R_{c} R_{y} R_{b}-R_{c} R_{y b}=-R_{c} L_{b y}$ $+R_{c} L_{y} L_{b}$. Using (2.a) this implies $R_{c} L_{y} L_{b} \in H$ or

(2.c) $R_{c} L_{c} L_{b}, R_{c} L_{b} L_{b} \in H$.

From (2.3), $(c w, y, b)=-(b, y, c w)$, we also have $L_{c} R_{y} R_{b}-L_{c} R_{y b}=-L_{c} L_{b y}$ $+L_{c} L_{y} L_{b}$. Using (2.b) this gives $L_{c} L_{y} L_{b} \in H$ or

(2.d) $L_{c} L_{c} L_{b}, L_{c} L_{b} L_{b} \in H$.

If we take $w=x=y$ in (2.4) and apply (2.2), we obtain

$$
\left(y, y^{2}, z\right)=(y, y, z y)+(y, y, z) y .
$$

Taking $x=y=z$ in (2.4) and applying (2.3), we also have

$$
\left(z^{2}, z, w\right)=\left(z, z^{2}, w\right) \text {. }
$$

Now (2.8), (2.9), (2.7), and (2.3) together imply

$$
\left(b^{2}, b, x\right)=2(b, b, b x)=-2(x b, b, b),
$$

whence $2 L_{b} L_{b} L_{b}, 2 R_{b} R_{b} R_{b} \in H$ or

(2.e) $L_{b} L_{b} L_{b}, R_{b} R_{b} R_{b} \in H$.

From $(2.7),(b, b, b x)=(b, b, x) b$, we have $L_{b} L_{b^{2}}-L_{b} L_{b} L_{b}=\left(L_{b^{2}}\right) R_{b}$ - $L_{b} L_{b} R_{b}$. Since $(2.6)$ and $(2.3)$ imply $\left(b^{2}, x, b\right)=0$ or $\left(L_{b^{2}}\right) R_{b}=R_{b}\left(L_{b^{2}}\right)$, this last equation is equivalent to $L_{b} L_{b^{2}}-L_{b} L_{b} L_{b}=R_{b} L_{b^{2}}-L_{b} L_{b} R_{b}$. Using (2.e) we now have

(2.f) $L_{b} L_{b} R_{b}=L_{b} R_{b} L_{b}=R_{b} L_{b} L_{b} \in H$.

From (2.7) and (2.3) we next obtain $(x b, b, b)=(b x, b, b)$. If we again use (2.e), this gives

(2.g) $L_{b} R_{b} R_{b}=R_{b} L_{b} R_{b}=R_{b} R_{b} L_{b} \in H$.

From (2.1) and (2.3) we have $-\left(c, x, c^{\prime} b\right)+\left(c^{\prime}, b,[x, c]\right)=c^{\prime}(b, x, c)+\left(c^{\prime}, x, c\right) b$. Using (2.b) this yields

(2.h) $R_{c} L_{c}, R_{b} \in H$.

Then from (2.3), $\left(b, x c, c^{\prime}\right)=-\left(c^{\prime}, x c, b\right)$, using (2.h) we obtain 
(2.i) $R_{c} R_{c}, L_{b} \in H$.

From (2.1) and (2.3) we also have $-\left(c, x, b c^{\prime}\right)+\left(b, c^{\prime},[x, c]\right)=b\left(c^{\prime}, x, c\right)$ $+(b, x, c) c^{\prime}$. Using (2.d) this yields

(2.j) $L_{c}, R_{c} L_{b} \in H$.

Then from (2.3), $\left(b, c x, c^{\prime}\right)=-\left(c^{\prime}, c x, b\right)$, using (2.j) we obtain

(2.k) $L_{c} L_{c}, R_{b} \in H$.

Linearization of (2.10) gives

$$
\begin{aligned}
\left(b^{2}, c, x\right)+(b c, b, x)+(c b, b, x) & =2[(b, b, c x)+(b, c, b x)+(c, b, b x)] \\
& =-2[(x b, b, c)+(x b, c, b)+(x c, b, b)] .
\end{aligned}
$$

If we now use (2.a) and (2.d), we have $2 L_{b} L_{c} L_{b}, 2 R_{b} R_{c} R_{b} \in H$ or

(2.m) $L_{b} L_{c} L_{b}, R_{b} R_{c} R_{b} \in H$.

Then from (2.3), $(b, c, b x)=-(b x, c, b)$ and $(b, c, x b)=-(x b, c, b)$, using (2.m) we obtain

(2.n) $L_{b} R_{c} R_{b}, R_{b} L_{c} L_{b} \in H$.

Next (2.4), (2.2), and (2.3) yield $(b c, x, b)=-(b, x, c) b$; while (2.1), (2.2), and (2.3) yield $(b c, x, b)+(b, c,[x, b])=-b(b, x, c)$. Subtracting the first of these equations from the second, we have $(b, c,[x, b])=-b(b, x, c)+(b, x, c) b$. Also, from $(2.1)$ one has $\left(b^{2}, x, c\right)+(b, b,[x, c])=b(b, x, c)+(b, x, c) b$. Adding this equation to the one just prior, we obtain $\left(b^{2}, x, c\right)+(b, b,[x, c])+(b, c,[x, b])$ $=2(b, x, c) b$. Using (2.c), (2.d), (2.m), and (2.n) this now gives $2 R_{c} L_{b} R_{b} \in H$ or

(2.p) $R_{c} L_{b} R_{b}=R_{c} R_{b} L_{b} \in H$.

From (2.4) and (2.3) we have $(c, x b, b)+\left(b^{2}, x, c\right)-(b, x, c) b+(b, b, c) x=0$. If we use (2.n) and (2.p), this yields

(2.q) $R_{b} L_{c} R_{b} \in H$.

Then (2.3), $(b, x b, c)=-(c, x b, b)$, using (2.q) gives

(2.r) $R_{b} R_{c} L_{b} \in H$.

From (2.4) and (2.3) we also have $(b, b x, c)+(c x, b, b)-(x, b, b) c+(c, x, b) b$ $=0$. If we use (2.b) and (2.q), this yields

(2.s) $L_{b} R_{c} L_{b} \in H$.

Then (2.3), $(b, b x, c)=-(c, b x, b)$, using (2.s) gives

(2.t) $L_{b} L_{c} R_{b} \in H$.

From (2.4) and (2.3) we next obtain $\left(x, b^{2}, c\right)-(x b, b, c)+(b, b, c) x-(x, b, c) b$ $=0$. Using $(2 \cdot \mathrm{m})$ this gives

(u.1) $R_{b c} R_{b} \in H$.

Again using (2.4) and (2.3) we have $(b, x c, b)-(b c, x, b)+(c, x, b) b-(b, c, b) x$ $=0$, that is $(b c, x, b)=(c, x, b) b$ using (2.2). If we use (2.b) and (2.q), this gives

(u.2) $L_{b c} R_{b} \in H$.

Lastly, (2.4) and (2.3) imply $(x, b c, b)+(b, b, x c)-(b, b, c) x+(b, c, x) b=0$. Using (2.c), (u.1), and (u.2) we have

(2.u) $L_{c} L_{b} R_{b}=L_{c} R_{b} L_{b} \in H$.

This completes the proof of the lemma. 
As in the case of generalized alternative algebras I, using Lemma 2.1 the following result now follows from the proof of Theorem 3 in [15].

Theorem 2.1. Let $A$ be a finite-dimensional generalized alternative algebra II over a field $F$ of characteristic $\neq 2$. If $B$ is a solvable subalgebra of $A$, then $B^{*}$ is nilpotent.

Corollary. Let $A$ be a generalized alternative algebra II over a field $F$ of characteristic $\neq 2$. If $x$ is a nilpotent element of $A$, then $R_{x}$ is nilpotent.

Lemma 2.2. Let $A$ be a generalized alternative algebra II over a field $F$ of characteristic $\neq 2$, and let $B$ be a subalgebra of $A$. If $x \in A$ is such that $x B \subseteq B, B x \subseteq B$, then $\left(x^{2} B\right) B \subseteq B, x^{2} B^{2} \subseteq B, B^{2} x^{2} \subseteq B, B\left(x^{2} B\right) \subseteq B$, and $\left(x^{2} B\right)^{2} B \subseteq B$.

Proof. We assume throughout that $b_{i} \in B$ for $i=1,2,3$. First using (2.4) and (2.2) we have $\left(x, b_{1} x, b_{2}\right)-\left(x, b_{1}, b_{2} x\right)-\left(x, x, b_{2}\right) b_{1}=0$ or $\left(x^{2} B\right) B \subseteq B$. Now from (2.4) we obtain $\left(x, x b_{1}, b_{2}\right)-\left(x, x, b_{2} b_{1}\right)+\left(x, x, b_{1}\right) b_{2}-\left(x, b_{1}, b_{2}\right) x=0$ or $x^{2} B^{2} \subseteq B$. Then (2.3), $\left(b_{1} b_{2}, x, x\right)=-\left(x, x, b_{1} b_{2}\right)$, gives $B^{2} x^{2} \subseteq B$. Next from (2.5) and (2.3) we have $\left(b_{1} b_{2}, x, x\right)=-b_{1}\left(x, x, b_{2}\right)-\left(x, x, b_{1}\right) b_{2}$ or $B\left(x^{2} B\right) \subseteq B$. Finally, (2.4), (2.2), and (2.3) give $\left(x, b_{1} x, b_{2}\right)-\left(x, b_{1}, b_{2} x\right)+\left(b_{2}, x, x\right) b_{1}=0$ or $\left(B x^{2}\right) B \subseteq B$. Since (2.4) and (2.2) yield $\left(x^{2}, b_{1}, x^{2} b_{2}\right)=\left(x^{2}, b_{1}, b_{2}\right) x^{2}$, this in turn gives $\left(x^{2}, b_{1}, x^{2} b_{2}\right) b_{3}=\left[\left(x^{2}, b_{1}, b_{2}\right) x^{2}\right] b_{3}$ in $\left(B x^{2}\right) B \subseteq B$. But then $\left[x^{2}\left[b_{1}\left(x^{2} b_{2}\right)\right]\right] b_{3}$ in $\left(x^{2} B\right) B \subseteq B$ implies $\left[\left(x^{2} b_{1}\right)\left(x^{2} b_{2}\right)\right] b_{3} \in B$, that is $\left(x^{2} B\right)^{2} B$ $\subseteq B$.

Using Theorem 2.1 and Lemma 2.2, the proof of the following theorem is now the same as the proof of Theorem 4 in [15].

Theorem 2.2. Let $A$ be a finite-dimensional generalized alternative algebra II over a field $F$ of characteristic $\neq 2$. If $A$ is a nilalgebra, then $A$ is nilpotent.

Theorem 2.3. Let $A$ be a simple, finite-dimensional, generalized alternative algebra II over an algebraically closed field $F$ of characteristic $\neq 2$. If $A$ has no nonzero idempotent other than 1 , then $A$ is itself a field.

Proof. Since $A$ a simple algebra implies $A^{2}=A, A$ cannot be nilpotent. Thus the finite-dimensionality of $A$ and Theorem 2.2 imply that $A$ is not a nilalgebra. Proposition 3.3 on p. 32 of [13] then ensures the existence in $A$ of a nonzero idempotent, which by assumption must be 1 . Now if characteristic $F=0$, from [6] it is known that $A$ is itself a field. On the other hand, if characteristic $F \neq 0$ and $A$ is not a field, then $A$ is a nodal algebra, that is $A=F 1+N$ where $N$ consists of nilpotent elements but is not a subalgebra of $A$. Now since from our earlier corollary we know that $x$ nilpotent implies $R_{x}$ nilpotent, it follows from Lemma 3 of [12] that $A$ cannot be nodal. Hence $A$ must be a field.

3. The Wedderburn principal theorem. Let $A$ be a power-associative algebra over a field $F$ of characteristic $\neq 2$ and define $x \circ y=\frac{1}{2}(x y+y x)$ for $x, y \in A$. If $A$ contains an idempotent $e$, then Albert has shown in [2] that $A=A_{1}+A_{1 / 2}$ 
$+A_{0}$ where $A_{i}=\{x \in A: x \circ e=i x\}$. In fact, $e x=x=x e$ for $x \in A_{1}$ and $e x=0=x e$ for $x \in A_{0}$. This decomposition of $A$ is known as the Albert decomposition.

Suppose now one also has $(A, e, e)=(e, A, e)=(e, e, A)=0$. If, as in the associative case, one takes $x=e x e+(e x-e x e)+(x e-e x e)+(x-e x-x e$ + exe), one sees that $A=A_{11}+A_{10}+A_{01}+A_{00}$ where $A_{i j}=\{x \in A: e x$ $=i x, x e=j x\}$. This further decomposition of $A$ is referred to as the Peirce decomposition.

Let $A$ be a generalized alternative algebra II over a field $F$ of characteristic $\neq$ 2. When $A$ contains an idempotent $e$, we will make use of the following results established by Kleinfeld in [5]:

(i) $I=(A, e, e)$ is an ideal of $A$ such that $I^{2}=0$.

(ii) If $A$ permits a Peirce decomposition, then for $i, j, k, t=0$ or 1 we have $A_{i j} A_{k t}=0$, when $j \neq k$, except for $A_{01} A_{01} \subseteq A_{10}$ and $A_{10} A_{10} \subseteq A_{01}$. Also $A_{i j} A_{j k} \subseteq A_{i k}$.

Lemma 2.3. Let $A$ be a generalized alternative algebra II over a field $F$. If $B$ is an ideal of $A$, then $A B^{2}+B^{2}=B^{2} A+B^{2}$ and $B^{3}$ are also ideals of $A$.

Proof. Throughout we assume $a, a_{i} \in A$ and $b_{i} \in B$ for $i=1,2,3$. Using (2.3) and the fact that $B$ is an ideal of $A$, we first observe that $\left(b_{1} b_{2}\right) a$ $=\left(b_{1}, b_{2}, a\right)+b_{1}\left(b_{2} a\right)=-\left(a, b_{2}, b_{1}\right)+b_{1}\left(b_{2} a\right)=-\left(a b_{2}\right) b_{1}+a\left(b_{2} b_{1}\right)+b_{1}\left(b_{2} a\right)$ implies $B^{2} A \subseteq A B^{2}+B^{2}$. Analogously one has $A B^{2} \subseteq B^{2} A+B^{2}$, and so $A B^{2}+B^{2}=B^{2} A+B^{2}$.

Now from (2.4), $\left(b_{1}, b_{2} a_{1}, a_{2}\right)-\left(b_{1}, b_{2}, a_{2} a_{1}\right)+\left(b_{1}, b_{2}, a_{1}\right) a_{2}-\left(b_{1}, a_{1}, a_{2}\right) b_{2}$ $=0$, we obtain $\left(B^{2} A\right) A \subseteq B^{2} A+B^{2}$; whence we have $\left(B^{2} A+B^{2}\right) A \subseteq B^{2} A$ $+B^{2}$. Next (2.4) and (2.3) together give $\left(a_{1}, a_{2} b_{1}, b_{2}\right)-\left(a_{1}, a_{2}, b_{2} b_{1}\right)+\left(a_{1}, a_{2}\right.$, $\left.b_{1}\right) b_{2}+\left(b_{2}, b_{1}, a_{1}\right) a_{2}=0$. Since we have just shown $\left(B^{2} A\right) A \subseteq B^{2} A+B^{2}$ $=A B^{2}+B^{2}$, we have $A\left(A B^{2}\right) \subseteq A B^{2}+B^{2}$; whence $A\left(A B^{2}+B^{2}\right) \subseteq A B^{2}$ $+B^{2}$. Thus $A B^{2}+B^{2}=B^{2} A+B^{2}$ is an ideal of $A$.

To show $B^{3}$ an ideal of $A$, one needs to show $\left[b_{1}\left(b_{2} b_{3}\right)\right] a,\left[\left(b_{1} b_{2}\right) b_{3}\right] a$, $a\left[b_{1}\left(b_{2} b_{3}\right)\right], a\left[\left(b_{1} b_{2}\right) b_{3}\right] \in B^{3}$. From (2.4), $\left(b_{1}, b_{2} a, b_{3}\right)-\left(b_{1}, b_{2}, b_{3} a\right)+\left(b_{1}, b_{2}\right.$, $a) b_{3}-\left(b_{1}, a, b_{3}\right) b_{2}=0$, we first obtain $\left[\left(b_{1} b_{2}\right) a\right] b_{3} \in B^{3}$ or $\left(B^{2} A\right) B \subseteq B^{3}$. This and (2.3) then give

$$
\left[\left(b_{1} b_{2}\right) a\right] b_{3}=\left[\left(b_{1}, b_{2}, a\right)+b_{1}\left(b_{2} a\right)\right] b_{3}=\left[-\left(a, b_{1}, b_{2}\right)+b_{1}\left(b_{2} a\right)\right] b_{3}
$$

or $\left(A B^{2}\right) B \subseteq B^{3}$. Similarly $\left[\left(b_{1} b_{2}\right) a\right] b_{3}=\left(b_{1} b_{2}, a, b_{3}\right)+\left(b_{1} b_{2}\right)\left(a b_{3}\right)=-\left(b_{3}, a\right.$, $\left.b_{1} b_{2}\right)+\left(b_{1} b_{2}\right)\left(a b_{3}\right)$ implies $B\left(A B^{2}\right) \subseteq B^{3}$, which with (2.3) in turn gives

$$
b_{3}\left[a\left(b_{1} b_{2}\right)\right]=b_{3}\left[-\left(a, b_{1}, b_{2}\right)+\left(a b_{1}\right) b_{2}\right]=b_{3}\left[\left(b_{2}, b_{1}, a\right)+\left(a b_{1}\right) b_{2}\right]
$$

or $B\left(B^{2} A\right) \subseteq B^{3}$.

We are now ready to show $B^{3}$ an ideal of $A$. Since we have just verified that $B\left(B^{2} A\right)$ and $\left(B^{2} A\right) B$ are contained in $B^{3}$, from $(2.1),\left(b_{1} b_{2}, b_{3}, a\right)+\left(b_{1}, b_{2},\left[b_{3}, a\right]\right)$ $=b_{1}\left(b_{2}, b_{3}, a\right)+\left(b_{1}, b_{3}, a\right) b_{2}$, we have $\left[\left(b_{1} b_{2}\right) b_{3}\right] a \in B^{3}$. This and (2.3) then give 
$\left[\left(b_{2} b_{3}\right) b_{1}\right] a=\left(b_{2} b_{3}, b_{1}, a\right)+\left(b_{2} b_{3}\right)\left(b_{1} a\right)=-\left(a, b_{1}, b_{2} b_{3}\right)+\left(b_{2} b_{3}\right)\left(b_{1} a\right)$ or that $a\left[b_{1}\left(b_{2} b_{3}\right)\right] \in B^{3}$. Next, since we have now shown $\left(A B^{2}\right) B$ and $A\left(B B^{2}\right)$ to be contained in $B^{3}$, from $(2.4)$ we obtain $\left(a, b_{1} b_{2}, b_{3}\right)-\left(a, b_{1}, b_{3} b_{2}\right)+\left(a, b_{1}, b_{2}\right) b_{3}$ $-\left(a, b_{2}, b_{3}\right) b_{1}=0$ or $a\left[\left(b_{1} b_{2}\right) b_{3}\right] \in B^{3}$. Finally, this and $(2.3)$ yield $a\left[\left(b_{2} b_{3}\right) b_{1}\right]$ $=-\left(a, b_{2} b_{3}, b_{1}\right)+\left[a\left(b_{2} b_{3}\right)\right] b_{1}=\left(b_{1}, b_{2} b_{3}, a\right)+\left[a\left(b_{2} b_{3}\right)\right] b_{1}$, whence $\left[b_{1}\left(b_{2} b_{3}\right)\right] a$ $\in B^{3}$. This completes the proof of the lemma.

Now, as in the case for standard algebras in [14], let $B$ be any ideal in $A$, a generalized alternative algebra II. We define $B^{\langle i\rangle}$ inductively by $B^{\langle 0\rangle}=B, B^{\langle i+1\rangle}$ $=A\left(B^{\langle i\rangle}\right)^{2}+\left(B^{\langle i\rangle}\right)^{2}$. By Lemma 2.3 this gives a descending chain $B^{\langle 0\rangle} \supseteq B^{\langle 1\rangle}$ $\supseteq \cdots \supseteq B^{\langle k\rangle} \supseteq \cdots$ of ideals of $A$ which we call a Penico sequence. We shall call $B$ Penico solvable in case there is some integer $k>0$ for which $B^{\langle k\rangle}=0$.

Lemma 2.4. Let $A$ be a generalized alternative algebra II over a field $F$. An ideal $B$ of $A$ is Penico solvable if and only if $B$ is solvable.

Proof. If $B$ is Penico solvable, then $B$ is clearly solvable since $B^{\langle i\rangle} \supseteq B^{(i)}$. On the other hand, suppose for any ideal $B$ of $A$ one has $B^{\langle 2\rangle} \subseteq B^{(1)}$. Then, as in the proof of Theorem 3 in [14], induction shows $B^{\langle 2 k\rangle} \subseteq B^{(k)}$, since $B^{\langle 2(k+1)\rangle}$ $=\left(B^{\langle 2 k\rangle}\right)^{\langle 2\rangle} \subseteq\left(B^{\langle 2 k\rangle}\right)^{(1)} \subseteq\left(B^{(k)}\right)^{(1)}=B^{(k+1)}$. Hence, if $B$ is solvable, then $B^{\langle 2 k\rangle}$ $\subseteq B^{(k)}=0$ for some $k$, that is $B$ is Penico solvable. Now by definition $B^{\langle 2\rangle}=A\left(A B^{2}+B^{2}\right)^{2}+\left(A B^{2}+B^{2}\right)^{2}$. Since $B^{3}$ is an ideal of $A$, to show $B^{\langle 2\rangle}$ $\subseteq B^{3}$ it suffices to verify $\left(A B^{2}+B^{2}\right)^{2}=\left(A B^{2}\right)\left(A B^{2}\right)+\left(A B^{2}\right) B^{2}+B^{2}\left(A B^{2}\right)$ $+B^{2} B^{2} \subseteq B^{3}$. But, since $B$ an ideal of $A$ implies that $A B^{2}$ and $B^{2}$ are contained in $B$, one has $\left(A B^{2}\right) B^{2}, B^{2}\left(A B^{2}\right)$, and $B^{2} B^{2}$ contained in $B^{3}$. Furthermore, since it has been demonstrated in the proof of Lemma 2.3 above that $\left(A B^{2}\right) B \subseteq B^{3}$, one has $\left(A B^{2}\right)\left(A B^{2}\right) \subseteq\left(A B^{2}\right) B \subseteq B^{3}$. Thus for any ideal $B$ of $A$ we have $B^{\langle 2\rangle} \subseteq B^{3} \subseteq B^{2}=B^{(1)}$, and the proof of the lemma is now complete.

Lemma 2.5. Let $A$ be a generalized alternative algebra II over a field $F$ of characteristic $\neq 2$. If $A$ contains an idempotent $e$, then the ideal $I=(A, e, e)$ satisfies $[A, I]=A_{1 / 2} I=\left(A_{1 / 2}\right)^{2} I=0$.

Proof. As observed in (i), Kleinfeld has shown $I$ to be an ideal of $A$ such that $I^{2}=0$. We also make use of the following observations. From (2.3) it follows that $(A, e, e)=I=(e, e, A)$. Thus (2.7) gives $(e, e, x)=(e, e, e x)+(e, e, x e)$ $=e(e, e, x)+(e, e, x) e$ or $I \subseteq A_{1 / 2}$. In particular, since (2.7) implies $e(e, e, x)$ $=(e, e, x) e$, we have

(2.v) $e k=\frac{1}{2} k=k e$ for $k \in I$.

Next let $(e, y, z)=a_{1}+a_{1 / 2}+a_{0}$ and $(e, e,[y, z])=b_{1 / 2}$ where $a_{i}, b_{i} \in A_{i}$ for $i=0, \frac{1}{2}, 1$. Then (2.1) yields $(e, y, z)+(e, e,[y, z])=e(e, y, z)+(e, y, z) e$ or $a_{1}$ $+a_{1 / 2}+a_{0}+b_{1 / 2}=a_{1}+e a_{1 / 2}+a_{1}+a_{1 / 2} e$, whence $a_{1}=a_{0}=b_{1 / 2}=0$ or

(2.w) $(e, e,[y, z])=0$,

(2.x) $(e, y, z) \in A_{1 / 2}$ for $y, z \in A$.

Since from [2] we know $y, z \in A_{1 / 2}$ implies $y \circ z \in A_{1}+A_{0}$, we also have $0=(e, e, y z+z y)+(e, e, y z-z y)=2(e, e, y z)$ or 
(2.y) $(e, e, y z)=0$ for $y, z \in A_{1 / 2}$.

Suppose now we are given $x \in A$. Let $x=x_{1}+x_{1 / 2}+x_{0}$ where $x_{i} \in A_{i}$ for $i=0, \frac{1}{2}, 1$. Then using (2.5), (2.3), (2.v), and (2.w) one has for $i=0,1$ and $k \in I$ that $x_{i} k=x_{i}(e, e, 4 k)+\left(e, e, x_{i}\right)(4 k)=4\left(e, e, x_{i} k\right)=4\left(e, e, k x_{i}\right)=(4 k)\left(e, e, x_{i}\right)$ $+(e, e, 4 k) x_{i}=k x_{i}$. Also using (2.y) and the fact that $I^{2}=0$, one has in addition that $0=\left(e, e, x_{1 / 2} k\right)=x_{1 / 2}(e, e, k)+\left(e, e, x_{1 / 2}\right) k=x_{1 / 2}(e, e, k)$ $=\frac{1}{4} x_{1 / 2} k$ as well as $0=\left(e, e, k x_{1 / 2}\right)=k\left(e, e, x_{1 / 2}\right)+(e, e, k) x_{1 / 2}=(e, e, k) x_{1 / 2}$ $=\frac{1}{4} k x_{1 / 2}$. Thus $[A, I]=0$ and, in particular, $A_{1 / 2} I=0$.

Next let $x, y \in A_{1 / 2}$ and $k \in I$. Then (2.1) gives $(x y, e, k)+(x, y,[e, k])$ $=x(y, e, k)+(x, e, k) y$. But $[A, I]=0$ implies $(x, y,[e, k])=0$, while $I$ an ideal of $A$ with $A_{1 / 2} I=0$ implies $x(y, e, k)=0=(x, e, k) y$. Hence $(x y, e, k)=0$. Let $x y=a_{1}+a_{1 / 2}+a_{0}$ where $a_{i} \in A_{i}$ for $i=0, \frac{1}{2}, 1$. Then $0=(x y, e, k)=[(x y) e] k$ $-\frac{1}{2}(x y) k=\left(a_{1}+a_{1 / 2} e\right) k-\frac{1}{2} a_{1} k-\frac{1}{2} a_{0} k=a_{1} k-\frac{1}{2} a_{1} k-\frac{1}{2} a_{0} k$, using the fact from [7] that for noncommutative Jordan algebras $A_{1 / 2} A_{i}, A_{i} A_{1 / 2} \subseteq A_{1 / 2}$ for $i=0,1$. Thus we have shown

(2.z) $(x y)_{1} k=(x y)_{0} k$ for $x, y \in A_{1 / 2}$ and $k \in I$.

Continuing as above we have $(e, x, y)=(e x) y-e(x y)=(e x) y-a_{1}-e a_{1 / 2}$. Since, by $(2 . \mathrm{x}),(e, x, y) \in A_{1 / 2}$, this gives $[(e x) y]_{1}=a_{1}$ and $[(e x) y]_{0}=0$. Then $a_{1}+a_{1 / 2}+a_{0}=x y=(e x) y+(x e) y$ implies $[(x e) y]_{1}=0$. Thus $(e x) y \in A_{1}$ $+A_{1 / 2}$ while $(x e) y \in A_{1 / 2}+A_{0}$. Now since from [7], as noted above, we know $x e \in A_{1 / 2}$, (2.z) gives $[(x e) y]_{1} k=[(x e) y]_{0} k$. But $[(x e) y]_{1}=0$, so $[(x e) y]_{1} k=0$ $=[(x e) y]_{0} k$. Hence $[(x e) y] k=[(x e) y]_{1} k+[(x e) y]_{1 / 2} k+[(x e) y]_{0} k=0$, since $A_{1 / 2} I=0$. In similar fashion we have $[(e x) y] k=0$. But then $x, y \in A_{1 / 2}$ gives $(x y) k=[(x e+e x) y] k=[(x e) y] k+[(e x) y] k=0$ or $\left(A_{1 / 2}\right)^{2} I=0$. This completes the proof of the lemma.

In proving the next theorem, we will use [8, Lemma 2.1, Theorem 2.1, and Theorem 2.2]. We make note that the exclusion in these results of characteristic 3 is not necessary [16].

Theorem 2.4 (Wedderburn principal theorem). Let $A$ be a finite-dimensional generalized alternative algebra II over a field $F$ of characteristic $\neq 2$, and let $N$ be the nil radical of $A$. If $A / N$ is separable, then $A=S+N$ (vector space direct sum) where $S$ is a subalgebra of $A$ such that $S \cong A / N$.

Proof. As in the proof of Theorem 23 on p. 47 of [1], it suffices to prove that $A$ contains a subalgebra $S \cong A / N$. Since our result is true trivially for $N=0$ or $N=A$, it is certainly true if $A$ has dimension one. We make an induction on the dimension of $A$ and assume the result true for algebras of dimension less than that of $A$.

From the proof of Theorem 23 on page 47 of [1], it now also follows that one may assume $N$ does not properly contain an ideal of $A$. Thus we may argue, as in the proof of Theorem 4 of [14], that $N^{2}=0$, for suppose $N^{\langle 1\rangle}=N$. Since $N$ is solvable by Theorem 2.2, $N$ is Penico solvable by Lemma 2.4. Hence $N=N^{\langle 1\rangle}=N^{\langle 2\rangle}=\cdots=N^{\langle k\rangle}=0$ for some $k$, and our result is immediate. 
Since, by Lemma $2.3, N^{\langle 1\rangle} \subseteq N$ is an ideal of $A$, we must then have $0=N\langle 1\rangle$ $=A N^{2}+N^{2}$, that is $N^{2}=0$.

At this point, an argument analogous to that used for Jordan algebras on page 289 of [3] shows one may also assume the field $F$ to be algebraically closed.

Suppose next that $A / N$ is not a simple algebra. If $B$ is a nodal subalgebra of $A / N$, then from [11] we know that $B$ has a homomorphic image which is a simple nodal algebra. Since our Theorem 2.3 denies this possibility, we have from Theorem 4 of [11] that $A / N$ semisimple implies $A / N=B_{1}+\cdots+B_{t}$ (algebra direct sum) where each $B_{i}$ is a simple ideal. Since Theorem 3 of [5] and our Theorem 2.3 imply each $B_{i}$ is alternative, each $B_{i}$ must have a unity element. Furthermore, from [7] we know $A$ a noncommutative Jordan algebra implies that $A_{1}$ and $A_{0}$ are subalgebras of $A$ for any idempotent $e \in A$. From Theorem 2.1 in [8] it now follows that it will suffice to consider the case $A / N$ a simple algebra.

As a final reduction we note, as in the proof of Theorem 2.2 of [8], that if there exists a primitive idempotent $e$ such that our result holds for the ideal $H$ generated by $A_{1 / 2}$, then it holds for $A$ as well.

Now $A / N$ not nil implies by Proposition 3.3 on p. 32 of [13] that $A / N$ contains a nonzero idempotent $e^{\prime}$. Should this be the only nonzero idempotent in $A / N$, then $e^{\prime}$ is a unit element for $A / N$, and Theorem 2.3 implies $A / N=F e^{\prime}$. By Lemma 2.1 in [8], $e^{\prime}$ lifts to an idempotent $e \in A$, and so we have $F e$ a subalgebra of $A$ such that $F e \cong A / N$. Hence we may assume that $A / N$ contains a nontrivial idempotent $e^{\prime}$. Again $e^{\prime}$ lifts to an idempotent $e \in A$. In particular, $e$ must be nontrivial and, since $A$ is finite-dimensional, one may assume that $e$ is primitive.

We now let $I=(e, e, A)$. By (i) and (2.3), $(e, e, A)=I=(A, e, e)$ is an ideal of $A$ such that $I^{2}=0$. Since, as earlier observed, we may assume $N$ not to properly contain an ideal of $A$, we must have either $I=0$ or $I=N$.

If we suppose first that $I=0$, then $A$ has a Peirce decomposition relative to $e$, since, by (2.2), $(e, A, e)=0$. Let $w_{i j}, x_{i j}, y_{i j}, z_{i j} \in A_{i j}$ for $i, j=0$ or 1 and consider $H=A_{10} A_{01}+A_{10}+A_{01}+A_{01} A_{10}$. Using the multiplication table described by (ii), it follows that to show $H$ an ideal of $A$ it suffices to show $A_{10} A_{01}$ an ideal of $A_{11}$ and $A_{01} A_{10}$ an ideal of $A_{00}$. Using (2.3) and the multiplication table described by (ii), one can compute as follows:

$$
\begin{aligned}
\left(x_{10} y_{01}\right) z_{11} & =\left(x_{10}, y_{01}, z_{11}\right)+x_{10}\left(y_{01} z_{11}\right) \\
& =-\left(z_{11}, y_{01}, x_{10}\right)+x_{10}\left(y_{01} z_{11}\right) \\
& =x_{10}\left(y_{01} z_{11}\right) \in A_{10} A_{01}
\end{aligned}
$$

and

$$
\begin{aligned}
z_{11}\left(x_{10} y_{01}\right) & =-\left(z_{11}, x_{10}, y_{01}\right)+\left(z_{11} x_{10}\right) y_{01} \\
& =\left(y_{01}, x_{10}, z_{11}\right)+\left(z_{11} x_{10}\right) y_{01} \\
& =\left(z_{11} x_{10}\right) y_{01} \in A_{10} A_{01} .
\end{aligned}
$$


Thus $A_{10} A_{01}$ is an ideal of $A_{11}$. Similarly one may show $A_{01} A_{10}$ to be an ideal of $A_{00}$, and hence $H$ is an ideal of $A$. In particular, $H$ must be the ideal generated by $A_{1 / 2}=A_{10}+A_{01}$.

Now $H$ a proper ideal implies by the induction hypothesis that our result is valid for $H$. Thus our final reduction applies, and we may conclude that our result is valid for $A$ itself. On the other hand, should $H=A$ then $A_{11}=A_{10} A_{01}$ and $A_{00}=A_{01} A_{10}$. Using (2.3) and the multiplication table described by (ii), we have

$$
\begin{aligned}
{\left[x_{11}\left(y_{10} z_{01}\right)\right] w_{11} } & =\left[-\left(x_{11}, y_{10}, z_{01}\right)+\left(x_{11} y_{10}\right) z_{01}\right] w_{11} \\
& =\left[\left(z_{01}, y_{10}, x_{11}\right)+\left(x_{11} y_{10}\right) z_{01}\right] w_{11}=\left[\left(x_{11} y_{10}\right) z_{01}\right] w_{11} \\
& =\left(x_{11} y_{10}, z_{01}, w_{11}\right)+\left(x_{11} y_{10}\right)\left(z_{01} w_{11}\right) \\
& =-\left(w_{11}, z_{01}, x_{11} y_{10}\right)+\left(x_{11} y_{10}\right)\left(z_{01} w_{11}\right)=\left(x_{11} y_{10}\right)\left(z_{01} w_{11}\right) \\
& =\left(x_{11}, y_{10}, z_{01} w_{11}\right)+x_{11}\left[y_{10}\left(z_{01} w_{11}\right)\right] \\
& =-\left(z_{01} w_{11}, y_{10}, x_{11}\right)+x_{11}\left[y_{10}\left(z_{01} w_{11}\right)\right]=x_{11}\left[y_{10}\left(z_{01} w_{11}\right)\right] \\
& =x_{11}\left[-\left(y_{10}, z_{01}, w_{11}\right)+\left(y_{10} z_{01}\right) w_{11}\right] \\
& =x_{11}\left[\left(w_{11}, z_{01}, y_{10}\right)+\left(y_{10} z_{01}\right) w_{11}\right] \\
& =x_{11}\left[\left(y_{10} z_{01}\right) w_{11}\right] .
\end{aligned}
$$

Since $A_{11}=A_{10} A_{01}$, these calculations show $A_{11}$ to be associative. Similarly one may show $A_{00}$ to be associative. If one then joins the calculations on p. 337 of [5], one may conclude that $A$ itself is an alternative algebra. But then from [9] our result is known to be valid for $A$, and the induction is complete.

Consider now the second alternative, namely $I=N$, and take $k=(e, e, x)$ $\neq 0$. We recall that, since $A$ is noncommutative Jordan, one has from [7] that $A_{1 / 2} A_{i}, A_{i} A_{1 / 2} \subseteq A_{1 / 2}$ for $i=0$, 1. In particular, this says that $N=I=(e, e, A)$ $\subseteq A_{1 / 2}$. Let $H$ be the ideal in $A$ generated by $A_{1 / 2}$, then $H=A_{1 / 2}+\left(A_{1 / 2}\right)^{2}$. To see this, let $x_{i}, y_{i}, z_{i} \in A_{i}$ for $i=0, \frac{1}{2}, 1$. Then for $i=0,1$ we have $\left(x_{1 / 2} y_{1 / 2}\right) z_{i}$ $=\left(x_{1 / 2}, y_{1 / 2}, z_{i}\right)+x_{1 / 2}\left(y_{1 / 2} z_{i}\right)=\left(x_{1 / 2}, y_{1 / 2}+z_{i}, y_{1 / 2}+z_{i}\right)-\left(x_{1 / 2}, y_{1 / 2}, y_{1 / 2}\right)$ $-\left(x_{1 / 2}, z_{i}, z_{i}\right)-\left(x_{1 / 2}, z_{i}, y_{1 / 2}\right)+x_{1 / 2}\left(y_{1 / 2} z_{i}\right)$ is in $N+\left(A_{1 / 2}\right)^{2} \subseteq A_{1 / 2}+\left(A_{1 / 2}\right)^{2}$, since $A / N$ simple implies as before that $A / N$ is alternative or that $(a, b, b) \in N$ for all $a, b \in A$. Similarly one has $z_{i}\left(x_{1 / 2} y_{1 / 2}\right) \in A_{1 / 2}+\left(A_{1 / 2}\right)^{2}$ for $i=0$, 1 . Since the cases for $i=\frac{1}{2}$ are immediate if one writes $x_{1 / 2} y_{1 / 2}=a_{1}+a_{1 / 2}+a_{0}$ where $a_{i} \in A_{i}$, we have $H=A_{1 / 2}+\left(A_{1 / 2}\right)^{2}$ as claimed. Now, by Lemma $2.5, H k=0$, while by (2.v) of Lemma $2.5 e k=\frac{1}{2} k \neq 0$. Thus, since $e \notin H, H$ is a proper ideal of $A$. Our final reduction now applies to complete the induction and the proof of the theorem.

In conclusion, the author would like to express his appreciation to Professor Erwin Kleinfeld under whose direction this research was accomplished. 


\section{BIBLIOGRAPHY}

1. A. A. Albert, Structure of algebras, Amer. Math. Soc. Colloq. Publ., vol. 24, Amer. Math. Soc., Providence, R. I., 1939. MR 1, 99.

2.—_ Power-associative rings, Trans. Amer. Math. Soc. 64 (1948), 552-593. MR 10, 349.

3. N. Jacobson, Structure and representations of Jordan algebras,Amer. Math. Soc. Colloq. Publ., vol. 39, Amer. Math. Soc., Providence, R. I., 1968. MR 40 \#4330.

4. E. Kleinfeld, Generalization of alternative rings. I, J. Algebra 18 (1971), 304-325. MR 43 \#308.

5.—, Generalization of alternative rings. II, J. Algebra 18 (1971), 326-339. MR 43 \#308.

6. E. Kleinfeld and L. A. Kokoris, Flexible algebras of degree one, Proc. Amer. Math. Soc. 13 (1962), 891-893. MR 25 \#5088.

7. K. McCrimmon, Structure and representations of noncommutative Jordan algebras, Trans. Amer. Math. Soc. 121 (1966), 187-199. MR 32 \#5700.

8. D. J. Rodabaugh, On the Wedderburn principal theorem, Trans. Amer. Math. Soc. 138 (1969), 343-361.

9. R. D. Schafer, The Wedderburn principal theorem for alternative algebras, Bull. Amer. Math. Soc. 55 (1949), 604-614. MR 10, 676.

10. - Noncommutative Jordan algebras of characteristic 0, Proc. Amer. Math. Soc. 6 (1955), 472-475. MR 17, 10.

11.— On noncommutative Jordan algebras, Proc. Amer. Math. Soc. 9 (1958), 110-117. MR 21 \#2677.

12.—, Restricted noncommutative Jordan algebras of characteristic p, Proc. Amer. Math. Soc. 9 (1958), 141-144. MR 21 \#2678.

13. - An introduction to non-associative algebras, Pure and Appl. Math., vol. 22, Academic Press, New York, 1966. MR 35 \# 1643.

14.—, Standard algebras, Pacific J. Math. 29 (1969), 203-223. MR 39 \# 5647.

15. - Generalized standard algebras, J. Algebra 12 (1969), 386-417. MR 44 \#268.

16. H. F. Smith, Ph.D. dissertation, University of Iowa, Iowa City, 1972.

Departient of Mathematics, University of Iowa, Iowa City, lowa 52242

Current address: Department of Mathematics, Madison College, Harrisonburg, Virginia 22801 